BULLETIN (New Series) OF THE

AMERICAN MATHEMATICAL SOCIETY

Volume 31, Number 2, October 1994

(C) 1994 American Mathematical Society

$0273-0979 / 94 \$ 1.00+\$ .25$ per page

Complements of discriminants of smooth maps: Topology and applications, by V. A. Vassiliev. American Mathematical Society, Providence, RI, 1992, 208 pp., \$164.00. ISBN 0-8218-4555-1

\title{
INTRODUCTION
}

This book is about diagonals and complements of diagonals together with their pervasive role in several branches of mathematics. Some of these mathematical areas include (1) algebraic functions, (2) complexity of algorithms, (3) knot theory, (4) topology of spaces of real functions without complicated singularities, and (5) classical homotopy theory. The spirit of the methods in this book has origins in the work of Arnold Shapiro, Wu Wen-Tsün, and Minoru Nakaoka, to name a few. Perhaps the main unifying point here is that useful and interesting consequences in the above subjects arise through an analysis of the complements of diagonals viewed through the eyes of cohomology groups. A few highlights of this book together with an attempt to explain some interrelations are given in this review. Several additions and corrections are included.

The ur-example of the principle of removing diagonals in algebraic topology is given by the classical Borsuk-Ulam theorem. Let the $n$-sphere, $S^{n}$, denote the set of points in Euclidean space $R^{n+1}$ of norm exactly one. If $x$ is a point in $S^{n}$, then $-x$ denotes the result of applying the antipodal map to the point $x$. A statement of the Borsuk-Ulam theorem is as follows:

If $h$ is any continuous function from $S^{n}$ to $R^{n}$, then there exists a point $x$ in $S^{n}$ such that $h(x)=h(-x)$ where $x$, of course, depends on $h$.

A classical method of proof for this theorem is given below, as the method provides a guide to a major point of view in this book. Let $X(n)$ denote the product $R^{n} \times R^{n}$ with the diagonal removed. There is a continuous function from the $n$-sphere to the product space $R^{n} \times R^{n}$ obtained by sending $x$ to the ordered pair $(h(x), h(-x))$. If the conclusion of the Borsuk-Ulam theorem were false, then this function $H$ takes values in the complement of the diagonal $X(n)$.

Furthermore, the function $H$ is equivariant in the sense that $H(-x)=$ $(h(-x), h(x))=\sigma H(x)$ where $\sigma$ permutes the coordinates of $X(n)$. Thus there is an induced continuous function from the quotient of the $n$-sphere by the action of $Z / 2 Z$ to the quotient of $X(n)$ by the analogous action of $Z / 2 Z$. The source space is homotopy equivalent to the real projective space of dimension $n$. The target space is homotopy equivalent to the real projective space of dimension $(n-1)$. Thus a contradiction to the existence of $H$ is obtained by inspecting its behavior in cohomology after "quotienting" by the natural action of the group of order two. The analysis of the complement of the diagonal together with the cohomology algebras of real projective spaces is the fuel which runs this theorem.

One might regard the above proof as instructive in at least two ways. The first way is that cohomology classes are obtained by quotienting out by the action of a group on a subspace of a product space where the group used above is 
$Z / 2 Z$. The analysis of these cohomology classes arises from the behavior of the complement of the diagonal in the product space. The second way is that useful information may be obtained for $k$-fold products with $k>2$.

The subspace of a $k$-fold product space $Y^{k}$ with all of the diagonals removed is an analogue of the case above where $k=2$ and also occurs ubiquitously in this book. This last construction is known to topologists as the (ordered) configuration space of $k$-tuples of distinct points in the space $Y$; see [FN]. The unordered configuration space is then the quotient by the symmetric group on $k$ letters acting by permuting coordinates. One particularly striking example of the configuration space occurs when $Y$ is the plane $R^{2}$. In this case, the unordered configuration space of $k$-tuples of distinct points in the plane has fundamental group which is isomorphic to Artin's braid group on $k$ strings, $B_{k}[\mathrm{FxN}]$. Furthermore, this space has trivial higher homotopy groups and is thus an Eilenberg-MacLane space of type $K\left(B_{k}, 1\right)$ [FN]. The next two sections provide beautiful applications of this $K\left(B_{k}, 1\right)$.

\section{Algebraic functions}

One of the subjects treated in this book arises from V. I. Arnol'd's study of entire complex functions [A1, A2, A3]. Consider the space $B(n)$ of all monic complex polynomials $p(z)=z^{n}+a_{n-1} z^{n-1}+a_{n-2} z^{n-2}+\cdots+a_{0}$ of degree $n$ which have no multiple roots. By identifying each such polynomial with its set of roots, one has that $B(n)$ is given by the space of unordered $n$ tuples of distinct points in the complex numbers $\mathbb{C}$. Thus $B(n)$ is precisely the configuration space of unordered $n$-tuples of distinct points in the plane and is a $K\left(B_{n}, 1\right)$.

Next fix a polynomial $p(z)$ as above, and assume that each of the $a_{i}$ 's are themselves complex polynomials in $k$ variables. Given such a polynomial, Arnol'd defines a function $\theta$ from $\mathbb{C}^{k}$ to $\operatorname{Sym}(\mathbb{C}, n)$ where this last space is the $n$-fold symmetric product, the $n$-fold product modulo the action of the symmetric group on $n$ letters $\Sigma_{n}$. Namely, for each $k$-tuple of complex numbers, there is a polynomial of degree $n$ which is obtained by substituting these $k$ complex numbers in the polynomial coefficients $a_{i}$ of $p(z)$. The resulting function is a polynomial in one variable of degree $n$ and thus has $n$ roots. The value of the algebraic function which Arnol'd defines, $\theta(p)$, at the $k$-tuple is then the unordered $n$-tuple of roots given above.

Let $D$ denote the subspace of $\operatorname{Sym}(\mathbb{C}, n)$ which contains all diagonals, and assume that $\theta$ takes on some value outside of $D$. Let $G$ denote the complement of the inverse image of $D, \theta^{-1}(D)$. Thus $G$ is an open complement of an algebraic hypersurface in $\mathbb{C}^{k}$. Arnol'd's map is the restriction of $\theta$ to a function $A(p): \mathbb{C}^{k}-G \rightarrow \operatorname{Sym}(\mathbb{C}, n)-D$. Observe that $\operatorname{Sym}(\mathbb{C}, n)-D$ is precisely the space $B(n)$ and that it can be regarded as a classifying space for these complex algebraic functions. Hence there are characteristic classes associated to $p$ given by pulling back the classes from the cohomology of the braid group.

One application of Arnol'd's construction of the function $A(p)$ is that certain entire algebraic functions are not given by superpositions of algebraic functions and polynomial functions in fewer variables. Namely, Arnol'd constructs specific algebraic functions $p(z)$ which "carry" non-vanishing cohomology classes by determining the maps $A(p)$ in cohomology (on certain classes in degrees one less than a power of two). He also proves that if $p(z)$ is decomposable by su- 
perpositions of functions in fewer variables, then these "characteristic classes" must vanish in the cohomology of $C^{k}-G$, the complement of a complex hypersurface. Thus these particular functions which Arnol'd constructs are indecomposable.

This beautiful method, although more technical to work out, is analogous to that of the classical Borsuk-Ulam theorem in which $A(p)$ is the analogue of the map $H$ given in Section 1. The main point is that the cohomology of the complement of certain diagonals is known together with their values or "characteristic classes" for the complex algebraic function under consideration above. Subsequently, more general results on the indecomposability of these complex algebraic functions were given in papers of [L]. A closely related application is presented next.

\section{COMPLEXITY OF ALGORITHMS}

Smale used the cohomology of the space $B(n)$, the space of monic complex polynomials of degree $n$ with no multiple roots, to study the topological complexity of algorithms for finding approximations to the zeroes of a complex polynomial $[\mathrm{Sm}]$. These results are described and extended in the book under review.

Consider the topological space $D(n)$ of all complex, monic polynomials of degree $n$ with all coefficients of norm bounded above by one. Fix a positive number $\varepsilon$. Smale poses problems concerning algorithms $P(n, \varepsilon)$ of determining all of the roots of polynomials in $D(n)$ to within $\varepsilon$. Vassiliev poses an analogous question, $P^{\prime}(n, \varepsilon)$, of determining one root to within $\varepsilon$. To study this question, Smale defined the topological complexity of algorithms for such problems $\tau(n)$; this measure is described in a crude way below.

An algorithm is a finite oriented tree with four types of nodes given as follows:

(1) a unique input node which accepts $2 n$ real numbers regarded as the real and imaginary parts of $n$ complex numbers of norm bounded above by one;

(2) computing nodes where, at each such node, certain real rational functions are computed from the input values together with values of similar functions evaluated at other computing nodes which occur before the one given in the algorithm;

(3) branching nodes where the value of one of the previously computed rational functions is compared to zero. Depending on whether their value is positive or negative, the control is then passed to one of two edges coming out of this node; and

(4) output nodes where, at each of these, the values of certain real rational functions in $2 n$ variables occurring earlier in the algorithm are declared to give the roots of a polynomial, and the program terminates.

An algorithm is defined to solve the problem $P(n, \varepsilon)$ if for any polynomial $q(z)$ in $D(n)$, (i) the corresponding execution path does not entail division by zero and (ii) there is an ordering of the roots of each polynomial $q(z)$ in $D(n)$ and the approximations for the roots in step (4) such that the norm of the differences of the $i$ th root and its $i$ th approximation is less than $\varepsilon$. The "topological complexity of an algorithm" is defined to be the number of branching nodes in it. The topological complexity of a problem $P(n, \varepsilon)$, denoted $\tau(n, \varepsilon)$, is the minimal topological complexity of algorithms which solve this problem. Define 
$\tau(n)=\lim _{\varepsilon \rightarrow 0^{+}} \tau(n, \varepsilon)$. There is an analogue $\tau^{\prime}(n, \varepsilon)$ for Vassiliev's generalization. Smale proved

$$
\tau(n) \geq\left(\log _{2} n\right)^{2 / 3}
$$

while Vassiliev improved this by a modification of the methods to

$$
n-1 \geq \tau(n) \geq n-\min D_{p}(n)
$$

where the minimum is taken over primes $p$ and $D_{p}(n)$ is the sum of the coefficients in the $p$-adic expansion of $n$.

The method of proof here is essentially an estimate of lengths of cup products in the cohomology of the complement of certain diagonals in a product space. Various generalizations to functions of several variables are also given.

\section{COHOMOLOGY OF KNOT SPACES}

Vassiliev also constructs invariants of knots which have stimulated attention, because these invariants contain the information given by the Jones polynomial as a special case. One survey is given in [BL].

These invariants are constructed as follows: Consider the space $K$ of all smooth maps from $S^{1}$ to $S^{3}$. Let the discriminant $\Sigma$ denote the subspace of $K$ given by maps with singularities or self-intersections. The main goal of this section is to obtain information about the zeroeth rational cohomology group of $K-\Sigma$, a group which distinguishes knot types.

First, Vassiliev gives finite-dimensional approximations to the knot space $K-\Sigma$. Consider the space of ordered triples of real, monic polynomials of degree $d+1$ with leading coefficient one, $\tilde{\Gamma}(d)$. Embed $\tilde{\Gamma}(d)$ in $\tilde{\Gamma}(w(d+1)-1)$ for $w>0$. Indeed Vassiliev specifically uses $w=3$. A small move of $\tilde{\Gamma}(d)$ in $\tilde{\Gamma}(3 d+2)$ leaves a new space $\Gamma(d)$ which is in general position with respect to the discriminant locus $\Sigma \cap \tilde{\Gamma}(3 d+2)$. The finite approximation for the knot space is then given by $\Gamma(d)-\Sigma$. Thus the next step is to analyze the reduced cohomology of these finite approximations to the knot space.

A second reduction is achieved by applying Alexander duality to $\Gamma(d)$, a method previously exploited in related problems [F, Se, BG]. Vassiliev then analyzes part of his spectral sequence which abuts the homology of $\Gamma(d)-\Sigma$. In addition to giving algorithms for computing these invariants together with their values, he gives other fascinating information about the cohomology of the knot space.

The invariants constructed in this chapter concerning knots have attracted much attention, and some of the points raised represent continuing work in progress. There are several programs in place to study the cohomology of these knot spaces, and the work given in this book is still very much in the process of development. Thus this review provides the briefest of sketches for this important subject.

There is a principle here; namely, various flavors of configuration spaces provide homological approximations for certain useful spaces. The spectral sequence here is an analogue of the Anderson spectral sequence (dating back to the 1970s) which is discussed in the next section.

\section{A PARTIAL SYNTHESIS}

The algebraic invariants used above arise from the cohomology of both the braid groups and configuration spaces. They also appear in a wider, more nat- 
ural setting through function spaces and have classical origins.

Consider the work of Shapiro [S] and Wu Wen-Tsün [W] in which they developed characteristic classes for embeddings of manifolds by considering product spaces with their diagonals removed. Inspection of their methods of course gives information about entire spaces of embeddings, although most of their work was focused on particular choices of embeddings. Write $\operatorname{Emb}(S, T)$ for the possibly empty space of continuous embeddings of $S$ into $T$. There is a map $\operatorname{Emb}(S, T) \times C(S, k) \rightarrow C(T, k)$ obtained by evaluating an embedding at a point in $C(S, k)$, the configuration space of ordered $k$-tuples of distinct points in $S$. Pull back cohomology classes from $C(T, k)$ to the product $\operatorname{Emb}(S, T) \times C(S, k)$, and evaluate them against a choice of class in the cohomology of $C(S, k)$ via a slant product. One then has a class in the cohomology of the space of embeddings. These invariants are non-trivial. Furthermore, they arise in the context of graded Lie algebras. Consider the example of the space of ordered embeddings of a discrete set with $k$ points in Euclidean $n$-space. The cohomology of this space was worked out for $n=2$ in [A1] and for any $n$ in [C1]. The result is that the cohomology is generated by classes $A(i, j)$ for $1 \leq j<i \leq n$ of dimension $(n-1)$. The relations are given by commutativity, associativity, and two others: (1) the square of $A(i, j)$ is zero and (2) the (three-term) relation $A(i, j) A(i, k)-A(j, k) A(i, k)+A(j, k) A(i, j)=0$. It was proven in [C] that these last two relations correspond, in a precise way, to (3) the anti-symmetry law and (4) the Jacobi identity in a graded Lie algebra. Indeed, the cohomology of the space of ordered embeddings can be identified with a stratification of the symmetric algebra generated by a graded free Lie algebra through the lens of the classical Poincaré-Birkhoff-Witt theorem $[\mathrm{Cl}$, $\mathrm{C} 2, \mathrm{CT}]$.

This story continues. Here let map ${ }_{*}(S, T)$ denote the space of continuous functions $f$ such that $f\left(s_{0}\right)=t_{0}$ for a choice of fixed base points $s_{0}$ in $S$ and $t_{0}$ in $T$. Thus if $S$ were $S^{n}$, the space $\operatorname{map}_{*}(S, T)$ would be the $n$-fold loop space of $T$ and would be written as $\Omega^{n} T$. One good feature of the $n$-fold loop space is that, with mild restrictions, its homotopy groups are isomorphic to those of $T$ with a degree shift.

These types of function spaces are frequently built up out of spaces like configuration spaces in a rather natural way. Consider the space of all functions of finite subsets of $R^{n}$ to $X$-\{point\}. If $X$ is a path-connected space satisfying certain mild topological restrictions, May proved that there is a map from this configuration space construction to the $n$-fold loop space of the $n$-fold suspension on $X$ which induces an isomorphism in homology or homotopy [Ma]. As does Segal, one might regard the above construction as a configuration space of points in $R^{n}$ with labels in $X$ [Se1].

Another basic example is a theorem of Segal regarding spaces of rational functions [Se2]. Let $\operatorname{Rat}_{q}\left(\mathbb{C} P^{n}\right)$ denote the space of ordered $(n+1)$-tuples of polynomials over $\mathbb{C}$ which are monic, of degree $q$, and do not vanish simultaneously. (Equivalently, $\operatorname{Rat}_{q}\left(\mathbb{C} P^{n}\right)$ is a space of unimodular rows.) Segal's result gives that these spaces are homotopy equivalent, through a range, to the space of all pointed continuous maps from the 2-sphere to the $(2 n+1)$-sphere, $\Omega^{2} S^{2 n+1}$. These spaces of rational functions fit together, and thus there is a limiting space of rational functions which, by Segal's theorem, is homotopy equivalent to $\Omega^{2} S^{2 n+1}$. The stable homotopy type of the spaces of rational 
functions was worked out in [CCMM], where their cohomology was also given; these cohomology groups are given in terms of that of the braid groups or $B(n)$. Some similar results are given in [K].

What about the cohomology of map $(S, T)$ for more general spaces $S$ ? Don Anderson gave a spectral sequence abutting the cohomology of this function space as long as $S$ is a manifold of dimension at most the connectivity of the target $T$ [An]. Of course, that $S$ is a manifold is not a significant restriction, as any finite complex has the homotopy type of a regular neighborhood of an embedding in a large Euclidean space. The $E_{2}$ term of Anderson's spectral sequence is given in terms of the cohomology of the configuration space of points in $S$ with labels in $T$. Anderson left research mathematics without publishing the details of his spectral sequence; a complete proof together with other information is given in the paper [BG]. Applications and features of this spectral sequence had already been recognized in the late 1970s in connection with Gelfand-Fuks cohomology and other subjects [CT]. Related constructions and results are given in [B, Mc].

Vassiliev also constructs this spectral sequence with several variations in this book where the "picture" is carried further. He reproves results in [CCMM] and [Se2] with these methods. A related example is given by Vassiliev's space of complements for discriminants of isolated singularities of holomorphic functions in $n$ complex variables. These spaces again fit together, and there is a stable "limit" version. A similar spectral sequence argument for these stable analogues gives that their cohomology is that of the $2 n$-fold loop of $S^{2 n+1}$. Yet another analogue is given by the stabilized version of "complements of caustics of holomorphic functions in $n$ complex variables"; the resulting cohomology ring is isomorphic to that of the $2 n$-fold loop space of the $2 n$-th suspension of the Grassmannian $U(n) / O(n)$. Another very interesting new example is given by the cohomology of spaces of real polynomials without multiple roots.

The homology of these types of function spaces naturally breaks apart into direct summands which themselves correspond to the associated graded modules obtained by filtering configurations by numbers of points. The resulting answers are given in terms of classical group (co)-homology. One can "read off" the homology of the braid groups, certain groups which are related to mapping class groups, or the homology of unordered configuration spaces directly from the homology of the iterated loop space of a sphere. Similar remarks apply to some ordered configuration spaces and explain the connection between their cohomology, a free Lie algebra, and Whitehead products in classical homotopy theory [BCT, BCM, C1, C2, C3]. Thus a fruitful point of view is that various function spaces provide a coherent and uniform picture of the types of invariants encountered in this book and in earlier work on these subjects.

Vassiliev's stimulating book does an admirable job of "stirring up the pot", throwing in new ideas, and giving interesting results. This book is worthwhile from many points of view.

\section{SOME CORRECTIONS AND ADDITIONS}

1. The cohomology of the braid group is given incorrectly in the statement on page 26. The correct answer for the stable braid group is listed here, while modifications for the other braid groups $B_{k}$ follow directly.

Let $p$ be an odd prime. The mod-p cohomology of the stable braid group 
is isomorphic to a tensor product of exterior algebras with generators in degree $2 p^{i}-1$ for $i$ at least zero and divided power algebras with generators in degree $2 p^{i}-2$ for $i$ at least one. The difference is that between a truncated polynomial algebra as stated on page 26 and a divided polynomial algebra. A similar result applies at $p=2$ where the cohomology is a tensor product of divided polynomial algebras with generators in degree one less than a power of two.

2. The mod- $p$ homology of the infinite symmetric group infinity was first determined in the 1950s by Nakaoka, who also determined the Hopf algebra structure $[\mathrm{N}]$. He also gave the cohomology ring structure. Nakaoka's results here formed a template for many further results in the subject, and they are analogous to the Araki-Kudo and Dyer-Lashof operations [AK, DL].

3. The homology of the braid group or configuration spaces of points in any Euclidean space with coefficients in either (1) the trivial representation, (2) the sign representation, or (3) a Coxeter representation was probably first given in [ $\mathrm{Cl}$, Section 4], where the "answers" are given by the interplay between free Lie algebras and these representations, although the language differs from that in Vassiliev's book. For example, the homology with coefficients in the sign representation is obtained by setting $X$, in the above reference, equal to a circle and shifting degrees. Similar results apply to other manifolds [BCT].

4. Vassiliev incorrectly states that his Theorem $16^{\prime}$ is a generalization of the main theorem in [CCMM]; indeed, Theorem $16^{\prime}$ is already given as Theorem 1.5 of that paper.

\section{REFERENCES}

[An] D. W. Anderson, A generalization of the Eilenberg-Moore spectral sequence, Bull. Amer. Math. Soc. 78 (1972), 784-788.

[AK] S. Araki and T. Kudo, The topology of $H$-spaces and $H$-squaring operations, Mem. Fac. Sci. Kyushu Univ. Ser. A (1956), 85-120.

[A1] V. I. Arnol'd, The cohomology ring of the colored braid group, Mat. Zametki 2 (1969), 247-248.

[A2] - On a class of algebraic functions and cohomology of swallow tails, Uspekhi Mat. Nauk 23 (1968), 247-248.

[A3] Topological invariants of algebraic functions, Funktsional Anal. i Prilozhen 4 (1970), 1-9; English transl. in Functional Anal. Appl. 4 (1970).

[BG] M. Bendersky and S. Gitler, The cohomology of certain function spaces, Trans. Amer. Math. Soc. 326 (1991), 423-440.

[BL] J. Birman and X. S. Lin, Knot polynomials and Vassiliev's invariants, Invent. Math. 111 (1993), 225-270.

[B] C.-F. Bödigheimer, Stable splittings of mapping spaces, Algebraic Topology (H. Miller and D. Ravenel, eds.), Lecture Notes in Math., vol. 1286, Springer-Verlag, New York, 1987, pp. 174-187.

[BCM] C.-F. Bödigheimer, F. R. Cohen, and R. J. Milgram, Truncated symmetric products and configuration spaces, Math. Z. 214 (1993), 179-286.

[BCT] C.-F. Bödigheimer, F. R. Cohen, and L. R. Taylor, On the homology of configuration spaces, Topology 28 (1989), 111-123.

[C1] F. R. Cohen, The homology of $\mathscr{C}_{n+1}$ spaces, Lecture Notes in Math., vol. 533, SpringerVerlag, New York, 1976, pp. 207-351; Bull. Amer. Math. Soc. 79 (1973), 761-764, 1236-1241. 
Artin's braid groups, classical homotopy theory and sundry other curiosities, Braids (Joan S. Birman and Anatoly Libgober, eds.), Contemp. Math., vol. 78, Amer. Math. Soc., Providence, RI, 1988, pp. 167-206.

[C3] The hyperelliptic mapping class groups $\mathrm{SO}(3)$ and $\operatorname{Spin}^{c}(3)$, Amer. J. Math. 115 (1993), 389-434.

[CCMM] F. R. Cohen, R. Cohen, B. Mann, and J. Milgram, The topology of spaces of rational functions and divisors of surfaces, Acta Math. 166 (1991), 163-221.

[CT] F. R. Cohen and L. R. Taylor, Computations of Gelfand-Fuks cohomology, the cohomology of function spaces, and the cohomology of configuration spaces, Lecture Notes in Math., vol. 657, Springer-Verlag, New York, 1978, pp. 106-143.

[DL] E. Dyer and R. Lashof, Homology of iterated loop spaces, Amer. J. Math. 84 (1962), 35-88.

[FN] E. Fadell and L. Neuwirth, Configuration spaces, Math. Scand. 10 (1962), 111-118.

[FxN] R. Fox and L. Neuwirth, The braid groups, Math. Scand. 10 (1962), 119-126.

[F] D. Fuks, Cohomology of the braid groups mod 2, Funktsional. Anal. i Prilozhen. 4 (1970), 143-151; English transl. in Functional Anal. Appl. 4 (1970).

[K] Y. Kamiyama, The modulo 2 homology groups of the space of rational functions, Osaka J. Math. 28 (1991), 229-242.

[L] V. Ya Lin, Artin's braids and related groups and spaces, J. Soviet Math. 18 (1979), 159227.

[LM] P. Löfler and J. Milgram, The structure of deleted symmetric products, Braids (Joan S. Birman and Anatoly Libgober, eds.), Contemp. Math., vol. 78, Amer. Math. Soc., Providence, RI, 1988, pp. 415-424.

[Ma] J. P. May, The geometry of iterated loop spaces, Lecture Notes in Math., vol. 268, Springer-Verlag, New York, 1972.

[Mc] D. McDuff, Configuration spaces of positive and negative particles, Topology 14 (1975), 91-107.

[N] M. Nakaoka, Homology of the infinite symmetric group, Ann. of Math. (2) 73 (1961), 229-257.

[Se1] G. Segal, Configuration spaces and iterated loop spaces, Invent. Math. 21 (1973), 213221.

[Se2] - Topology of spaces of rational functions, Acta. Math. 143 (1979), 39-72.

[S] A. Shapiro, Obstructions to the imbedding of a complex in a euclidean space, I. The first obstruction, Ann. of Math. (2) 66 (1957), 256-269.

[Sm] S. Smale, On the topology of algorithms. I, J. Complexity 3 (1987), 81-89.

[V] F. V. Vainshtein, Cohomology of the braid groups, Funktsional. Anal. i Prilozen. 12 (1978), 72-73; English transl. in Functional Anal. Appl. 12 (1978).

[W] Wu Wen-Tsün, $A$ theory of imbedding, immersion, and isotopy of polytopes in a Euclidean space, Science Press, Beijing, 1974.

FREDERICK R. COHEN

UNIVERSITY OF ROCHESTER

E-mail address: cohf@db1.cc.rochester.edu 